\title{
A Knowledge-Based Decision Support System For Costing Job Orders
}

Lakshminarayana Talluru, (E-mail: Talluru@hotmail.com), Intel Corporation Ashutosh Deshmukh, (E-mail: avd1@psu.edu), Pennsylvania State University, Erie

\begin{abstract}
The problem of bidding on a customized product in a manufacturing environment is very complex. Such bidding requires determination of labor, material, production process requirements, outsourcing strategies, if necessary, and the recognition of profit margins. The purpose of this paper is to describe the development of knowledge based decision support system to deal with the bidding problem for a forging and steel company. The decision support system captures engineers' expertise, uses group technology principles for product classification, and uses the business domain specific knowledge. It is implemented in Microsoft Access, a relational database system. The objectives of this decision support system were to cost the majority of the jobs efficiently and effectively, standardize the bidding process, aid the salespersons, and provide means to facilitate learning from past successful and unsuccessful bids. The formidable problems encountered in development, validation, and implementation of the system are also discussed.
\end{abstract}

\subsection{Introduction}

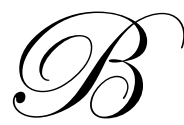

uilding a customized product in a manufacturing environment poses many challenges. The primary hurdle is the ability of the company to efficiently and effectively bid on the jobs. A customer's decision to accept or reject a bid is usually based on how confident he/she feels that the company submitting the bid has the capability to timely deliver the quality work at the appropriate price. The bidding company's chances of success on these measures are enhanced by increasing the reliability of quotes. However, in a customized job order products, the timeliness of the quote and its accuracy are inversely related.

In the manufacturing environment the bidding problem is more complex than merely quoting a cost estimate to the customer. Complex custom orders can have numerous manufacturing steps, which may require detailed modeling of the problem. Such modeling involves determining the labor, material, production process requirements, outsourcing strategies, if necessary, and the recognition of profit margins. This process is expert intensive and may take several days of an expert engineer's time, precluding a salesperson from creating a bid on the site.

The bidding process on customized orders is computationally intensive, requires expertise from engineers, and is ill structured. The bidding process is also expensive, the acceptance rate of bids is generally low, and any inefficiencies in the process can cause substantial costs to the company. Expert systems and knowledge based decision support systems are normally used in such situations (Edwards, Duan, and Robins, 2000). These systems have been used in a variety of problem settings and are expected to support, augment, or automate decision making. A scorecard for these systems is mixed, in some cases expert systems and decision support systems have been beneficial and in some cases - complete failures (O Keefe and Preece, 1996).

The application of knowledge based decision support system to cost estimation and pricing decisions has been explored by Kingsman and De Souza (1997). In this paper, the authors thoroughly discuss the conceptual underpinnings of cost estimation and pricing in engineered-to-order and make-to-order environments. They discuss process model for customer enquiries, nature of rules used for estimation and pricing, decision maker's biases, and

Readers with comments or questions are encouraged to contact the authors via email. 
identification of heuristic expert rules. However, our review of the literature failed to elicit a study that described the development of a decision support system for cost estimation and pricing decision in an engineered-to-order or make-to-order environments. We describe the development of the decision support system for a specific company by utilizing group technology, relational database, and VBA programming.

The purpose of this paper is to demonstrate the application of a knowledge based decision support system to a bidding problem in a manufacturing environment. This decision support system was created for a forging and steel company to deal with various problems associated with the bidding of customized products. The objectives of this decision support system were to cost the majority of the jobs effectively and efficiently, standardize the bidding process, capture the expertise of engineers, aid the salespersons, and provide a means to facilitate learning from past successful and unsuccessful bids. The decision support system was developed by capturing engineers' expertise, group technology coding for product classification, and implemented through a relational database system utilizing Microsoft Access. The complexity of the system caused severe testing and validation problems, which are also discussed.

\subsection{The Bidding Process}

The corporation is involved in producing customized products such as ship shafts, rolls, rotors, etc. for commercial and defense purposes. The shapes of these products are shown in Figure 1.

\section{Figure 1}

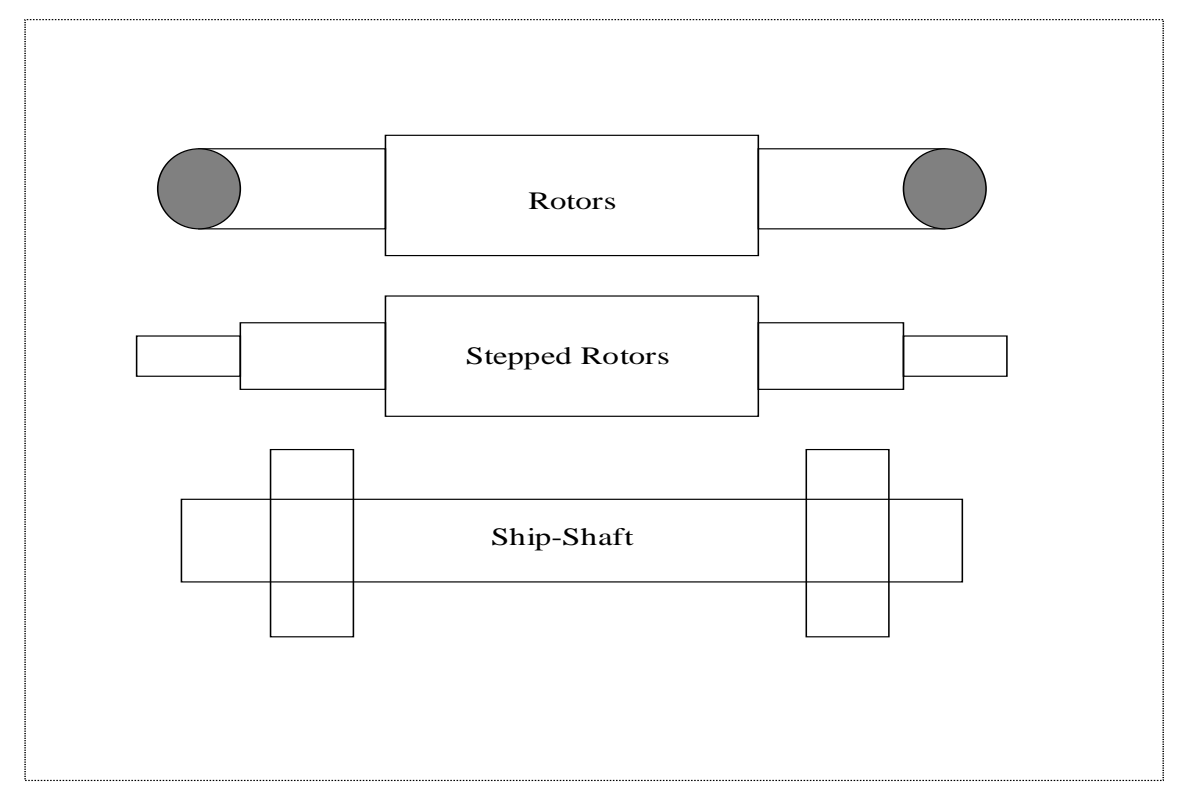

The company develops blueprints for each order or occasionally the customer supplies the blueprints. The first step in the bidding process is to identify the product given the feature set. It includes comparing the product with former products and determining whether the company created an identical or similar product earlier. In the majority of the cases the new order is similar (not identical) to some earlier product. In very exceptional (approximately 5\%) cases the product is entirely new and has no earlier frame of reference, the decision support system is not designed to handle these exceptional cases. This process of product identification used to be a manual process and consumed substantial time of the personnel involved in identification and retrieval mission. 
Once the product is identified then the issue of labor costs, material costs, manufacturing costs, and profits margins is handled. This issue is expert intensive and more complex compared to the costing of mass scale production. In the later situation, the product design is standardized, the manufacturing operations are well researched, and the known costs are identified. In a job order situation, the business may not have control on some of these issues such as the ability to standardize the product design or satisfying all the customer requirements.

The primary cost driver for labor, material, and manufacturing costs is the steps involved in the production process. The production process involves the following steps:

- $\quad$ Selecting appropriate steel ingot

- $\quad$ Shaping the ingot using a variety of operations such as heat treatments, machining, or forging

- $\quad$ Routing of the ingot through the shop floor and using different machines for shaping depending on the choice of heat treatments, machining, or forging. These operations are not mutually exclusive and two or more operations can be used for the same purpose, however, the costs of these operations differ.

Technically, no two jobs are identical. However, products are usually similar in terms of shape and processing requirements, while differing in technical specifications such as size, materials, or machining tolerances. The manufacturing processing time, an important cost element, is generally calculated using mathematical formulas based on these technical specifications.

The human experts, engineers, use their experience to assign the right numbers for cost assessment. For example, a particular job may cost more or less than usual depending on the type of ingot used for this product or the number of heats required by the material specifications. The engineers also look at the labor requirements and the associated costs. Some of these issues cannot be captured using mathematical formulas. In selecting an ingot to manufacture the product, or estimating manufacturing process time, the engineers will bring their expertise to this process and that may not be capable of formula expression. Finally, the profit margin for the job needs to be determined. The profit margin sometimes was a standard percentage or sometimes engineers used other formulas. The concern, of course, is to recover direct costs, contribution to overhead; and appropriate profit.

The proposed decision support system should be capable of supporting salespersons in the field and should enable engineers to input and update the knowledge base. Theoretically the functions of the decision support system will be:

- Identify or help in identifying the new product.

- Identify the ingots that are suitable for the job.

- Identify the production process such as routing through the plant.

- $\quad$ Suggest the profit margin for the job or allow inputting of numbers.

- Develop a knowledge base to store the knowledge concerning the past products, the expertise acquired from the engineers about the generic products such as formulas and heuristic rules to calculate process times for each machine/operation combination, and the associated costs.

- $\quad$ Provide the information to salespersons in real time, and allow updating of the knowledge base.

- $\quad$ Be user friendly.

\subsection{Application of Group Technology}

The first step involved in the bidding process is classification of the customer specified product based on comparison with the earlier products manufactured by the company. We employ a modified group technology coding procedure that classifies products based on similarity in feature sets and processing requirements in conjunction with the other customer requirements.

Group technology is a method of classifying products (interchangeably used with the term "parts") or manufacturing processes based on common characteristics. These characteristics include part size, part geometry, routing requirements, manufacturing tolerances, among other things. Jakubowski (1982), Henderson (1984), Li 
(1988), and Ben-Arieh, Lee, and Chang (1996) developed classification schemes based on part geometry. Group technology has also been applied to various aspects of product design and manufacturing (Djassemi, 2000). Since group technology proposes common solutions to similar problems, it can be applied to a wide range of problems. For example, rapid retrieval of design data (Dowlatshai and Nagraj, 1998), forming part families and machine cells (Cheng, Goh, and Lee, 2001), and classification of part families based on images (Kuo, Chi, and Teng, 2001).

Wemmerlov and Hyer (1986), Vakharia (1986), and Gunasekaran, Goyal, Virtanen, and Yli-Olli (1994) in the review of group technology coding procedures indicate that the existing research on part classification procedures tends to be very abstract and difficult to apply in practice. However, if the group technology coding is properly used then it is a powerful tool to identify products.

Billo and Bidanda (1995) show common basis for several object-oriented modeling principles with those used in coding and classification for group technologies. Their objective is to establish a hierarchical set of subclassifications that will uniquely define each product. The object-oriented paradigm provides and effective means of organizing information about both the product and the manufacturing process. We employ this object-oriented paradigm to group technology for part classification and identification scheme.

Consider the example of "rotors." As shown in Figure 2, different types of rotors can be classified using a hierarchical classification. Rotors can broadly be classified as "electrical" or "locomotive." Under electrical category, a further classification can be made as "hydro" or "thermal" and so on. A group technology coding system can now be developed based on this hierarchy and may need three numbers to capture the three hierarchies. For example, a code of 11 may represent rotor - electrical, and a code of 12 may represent, rotor - locomotive. If we define "rotor" as an object then "electrical" and "locomotive" categories will inherit the properties of "rotor" object. We simulated such object properties in Microsoft Access by defining each "part" as a table.

However, the customer often in addition to the type of product may specify materials required, for example, a specific type of steel out of thousands of types, heat treatments, and specific tests before the product is delivered. We modified the group technology code by adding additional codes that captured customer requirements. This system efficiently captures prior products each one of may differ from other slightly or severely.

The modified group technology coding system, in the context of this study, can help the user in answering the question "has this ship shaft (or a similar) item been made before?" The application of group technology must be at such a level as to allow salespersons with limited technical training to translate traditional customer requirements into a group technology code that can be used to recognize existing manufacturing processes and costs and apply formulas to generate competitive bids. The modified group technology coding developed for the system works well in this respect.

\subsection{Architecture of the Decision Support System}

The architecture for the proposed decision support system (called XpertBid) is shown in Figure 3.

The system operates in two environments: 1) development environment and 2) consulting environment. The development environment enables engineers to manipulate the requirements (such as ingot specifications and manufacturing processes) for existing products or design requirements for new products. The consulting environment, on the other hand, permits sales staff in preparing bids for the jobs. Both of these environments are now described sequentially.

The human experts, engineers, participate in the development environment. The decision support system acquires the needed expertise from the engineers using intelligent user interface. Knowledge base consists of all the information required for product classification. In practice, the information associated with each product must be entered once by the expert engineer. The information set consists of definition of the product feature set, the required dimension (large versus small), the computation of forging and heat allowances, ingot selection, and specifying the expected routing through the plant (depending on whether the product is bored or solid, step bored or straight bored, etc.). Additionally, any new manufacturing processes must be defined along with the supporting manufacturing process time formulas. 
Figure 2

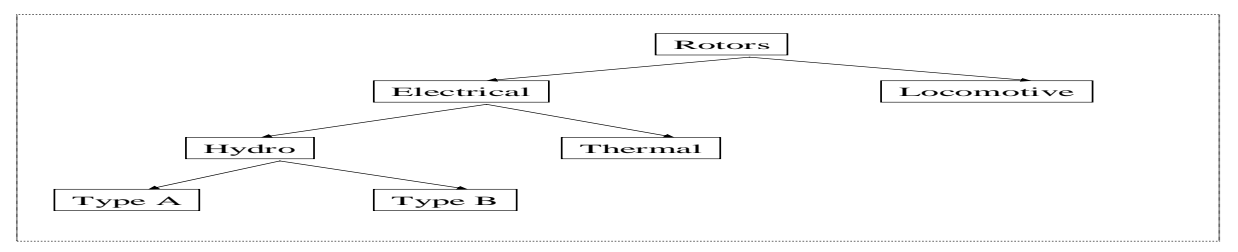

Figure 3

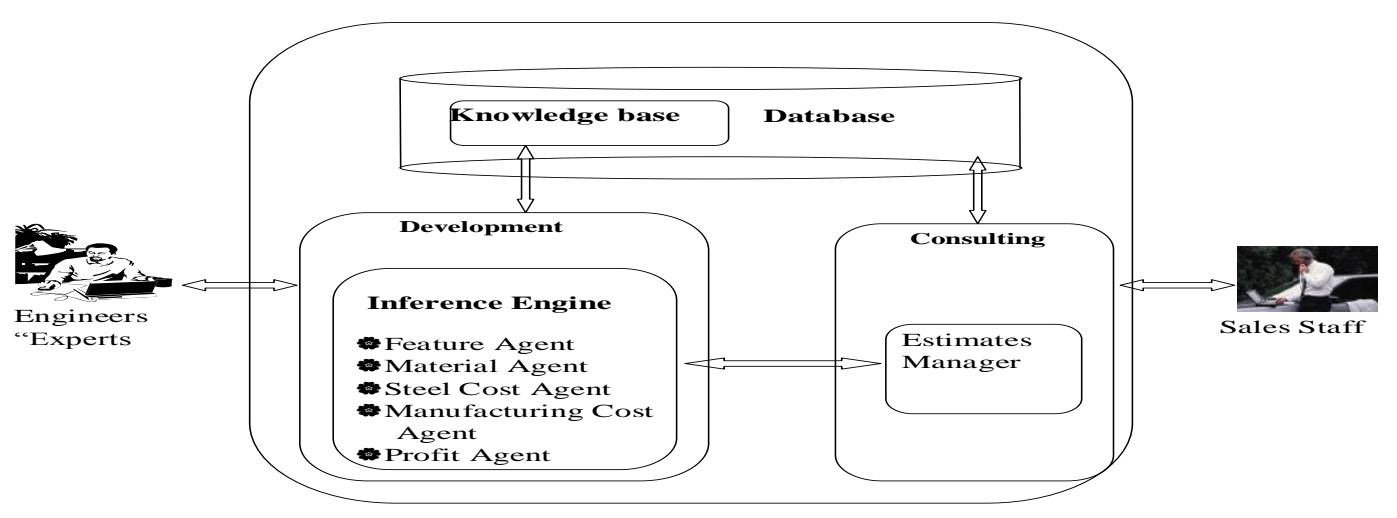

This knowledge base (and also the transactional data such as log of all bids) is stored in the Microsoft Access environment as a variety of tables and event procedures. The software was chosen for user friendliness, database features, and the programming language Visual Basic for Applications (VBA) that can be used for simulated object oriented programming facilities. The knowledge base two components: declarative and procedural. The characteristics of feature set, dimensionality, etc. are stored as a declarative knowledge. On the other hand, computation of forging or heat allowances, ingot selection, and occasionally manufacturing processes such as routing of the ingot through the plant requires rules. For example, the rule base for computation of heat treatment allowances is shown in Table 1. The rules are based on length and diameter of the selected ingot. The rule base at the physical level is stored as decision tables since it is easier to maintain rules as tables in the database environment. If the rule base includes calculations using formulas then these procedures are stored using VBA. The entire knowledge base consists of approximately 50 tables, $200 \mathrm{VBA}$ routines (that includes calculations, controls, and user interfaces), and approximately 75 rules. The company had dealt with 600 customized products and our knowledge base captured around 40 of those products. 
Table 1 Rule Base for Heat Treatment Allowances

\begin{tabular}{|c|c|c|c|c|c|}
\hline ID & Min. D & Max. D & Min. L & Max. L & Heat Treatment Allowance \\
\hline 1 & 0 & 20 & 0 & 0 & 0.500 \\
\hline 2 & 0 & 20 & 30 & 30 & 0.750 \\
\hline 3 & 0 & 999 & 40 & 40 & 1.000 \\
\hline 4 & 20 & 60 & 0 & 0 & 0.500 \\
\hline 5 & 20 & 30 & 10 & 10 & 0.625 \\
\hline 6 & 20 & 30 & 30 & 40 & 0.750 \\
\hline 7 & 30 & 60 & 10 & 30 & 0.750 \\
\hline 8 & 30 & 60 & 30 & 40 & 1.000 \\
\hline 9 & 60 & 999 & 0 & 10 & 0.750 \\
\hline 10 & 60 & 999 & 10 & 999 & 1.000 \\
\hline
\end{tabular}

Knowledge about the products at the logical level is represented as objects and each product is represented as a class structure. A subset of the object model is shown in Figure 4.

The product object includes such data attributes as product number, product description, product identification method, forge allowance methods, ingot selection methods, and various others. These methods are used to make certain important engineering decisions. For example, determination of forging allowances differs greatly depending upon the product class. The method used to demarcate the forging allowances for a rolling pin, shown in Figure 1, is not the same method used for a ship shaft item.

his engineering decision is critical. The forging process is inexpensive compared to the machining process employed to reduce an ingot to the necessary size of the requested product. However, there are size limitations and accuracy issues related with the forging process. For example, the product must be of certain minimum length to be forged.

The forging allowances are computed based on the largest diameter, also termed "body diameter," and the number of steps (or arms) required on the rolling pin. The decision to forge or to machine depends on the type of press used, length of the area, the amount of drop (or reduction), the shape of the product, and factors such as setup time and labor costs. This process was not only considered complex but also very time consuming. Similarly, the ingot selection is a subjective process. The general rule of thumb is to select the ingot with a sufficient body weight and mean diameter to produce the required part without generating too much waste. Once an ingot is used, the residual material is considered scrap and is sold at a minimal price. Thus, selecting a wrong ingot may result in dramatic increase in the overall costs for those jobs.

Each product has a unique router containing many operations. The sequence of these operations is captured using a sequence number that becomes part of the key set for the router, as shown in Figure 4 . The other objects in Figure 4 are self-explanatory. Operations can be carried out using more than one machine and machines are capable of handling many operations. Several machines may be capable of handling an operation in a given router. The machine selection may require additional expertise based on machine availability, costs, and other information. This expertise is also stored as tables or encoded in VBA as formulas. The tables are connected to each other through primary and secondary keys. For example, product table's primary key is Product Number, router table's primary key is Sequence Number and secondary key is Product Number, and so on. The object model shown in Figure 4 is capable of storing such an expertise.

The knowledge base regarding determination of profit margins consists of formulas, suggestions, and prompting for user input. If the product is already in the database then the profit margin is suggested by the system. If the product is different and/or complex then the system will suggest the involvement of engineers or senior sales management. The salespersons can also input appropriate profit margin based on consultation with management or their prior experience with that class of products and current market conditions. The decision support system has the discriminatory rules for all these situations stored as tables or formulas.

The inference engine, as shown in Figure 3, is responsible for carrying out the bidding process. It is divided into the following categories: the feature agent, the steel cost agent, the manufacturing cost agent, and the profit agent. The feature agent is responsible for product identification. The feature agent extracts the features from the user input and generates the product number based on the group technology coding. For example, product Billet 
has certain characteristics such as Billet type, Shape, Treatment, Steel Type, and Cut Type. Each characteristics has certain choices, the dominant choices are listed first and then the next dominant ones and so on. Each choice has a certain number associated with it; for example, three choices - Commercial Quality, Special Quality, and Forged Bar - under Billet Type may be associated with numbers 1,2, and 3. Based on the user input the system creates a product code and compares it with the past product knowledge, which is stored as group technology codes. If an identical product is found then the system will proceed with the bidding job. If an identical product is not found then the system will narrow down the choices of possible products. And the engineer will complete the product classification shortly thereafter. The system thus shortens the time period required for bidding quotes.

The material agent is responsible for determining the heat treatments and tests that are needed for the item. The customer typically dictates the material specifications. The heat treatment procedures and test codes influence both the material costs and the manufacturing costs. The steel cost agent is accountable for computing the material costs. This is the standard accounting procedure involving steel costs, scrap costs, shipping weights, forging weights, and so on. To compute these costs, the steel cost agent sends a message to the product object and obtains forging allowances, ship weights, forge weights, ingot used, and other needed information.

The manufacturing cost agent supervises the router operations. The actual computation of the manufacturing cost is a straightforward procedure. The actual cost is a function of the type of machine used and the time consumed on that machine. The major issue is the formula used to determine the operation processing time is stored as a data value in the database. As shown in Figure 3, the generic cycle time formula is a data attribute of the Operation object. The router object may overwrite it. The manufacturing cost agent monitors the validity of the formula in the context of the database.

Finally, the profit agent is responsible for determining the profit margins. For some products the rule may be as simple as adding $25 \%$ or $40 \%$ margin and for others it may be very subjective and is supervised by the senior engineers and sales management. The system also allows the salespersons to overwrite the margins and insert new profit margins. As already mentioned, the human experts, engineers, continuously update the rules for new products.

The sales staff uses the consulting environment to facilitate the bidding process. Typically, job orders are initiated by the customers through the sales staff. Job order manufacturers generally organize their sales staff based on product lines with the expectation that salespersons will become familiar with the particular products they sell. The sales staff seldom encounters particulars regarding detailed cost estimation steps when using the tool for its primary function. However, the rigorous process of translating customer requirements into a competitive bid provides a powerful diagnostic tool for evaluating both the bidding and manufacturing process.

The first user screen encountered in the consulting environment is "Estimate Manager." The Estimate Manager is a typical transaction processing system that accepts the customer inquiries and generates the bids. The sales staff begins the bidding process by entering the customer details. The salesperson can choose "Quote Type" as Steel or Component Items. If steel is chosen then product types under steel can be chosen by adding a line estimate on the Estimate Form. To add a line item on the estimate, the Estimate Manager provides the "Item Definition Main Menu" as shown in Figure 5.

Designed to meet the practitioner need, the form of the feature set is different from the input criteria of the most group technology coding procedures. Rather than using generic shape classifications such as rotational versus non-rotational, the sales people enter information by product (class) name, for example, billets, bars, etc. Figure 5 outlines the hierarchical framework used to classify customer requests. The features input by the user are used to determine product number. If necessary, the system will bring up another appropriate screen to capture additional details. Once the item details are entered, the system will come up with the unit price (sale price) for the product. All the information captured about the sales transaction is stored in the database.

It should be noted that the sales people are generally familiar with the description shown in Figure 5 . Moreover, it is natural for them to work through the hierarchy down to the lowest level (shown for the Steel Sales > 
Billets > Special or Standard) to input screens that facilitate recording the requested dimensions as well as the steel type and any other special requirements.

\section{Figure 4}

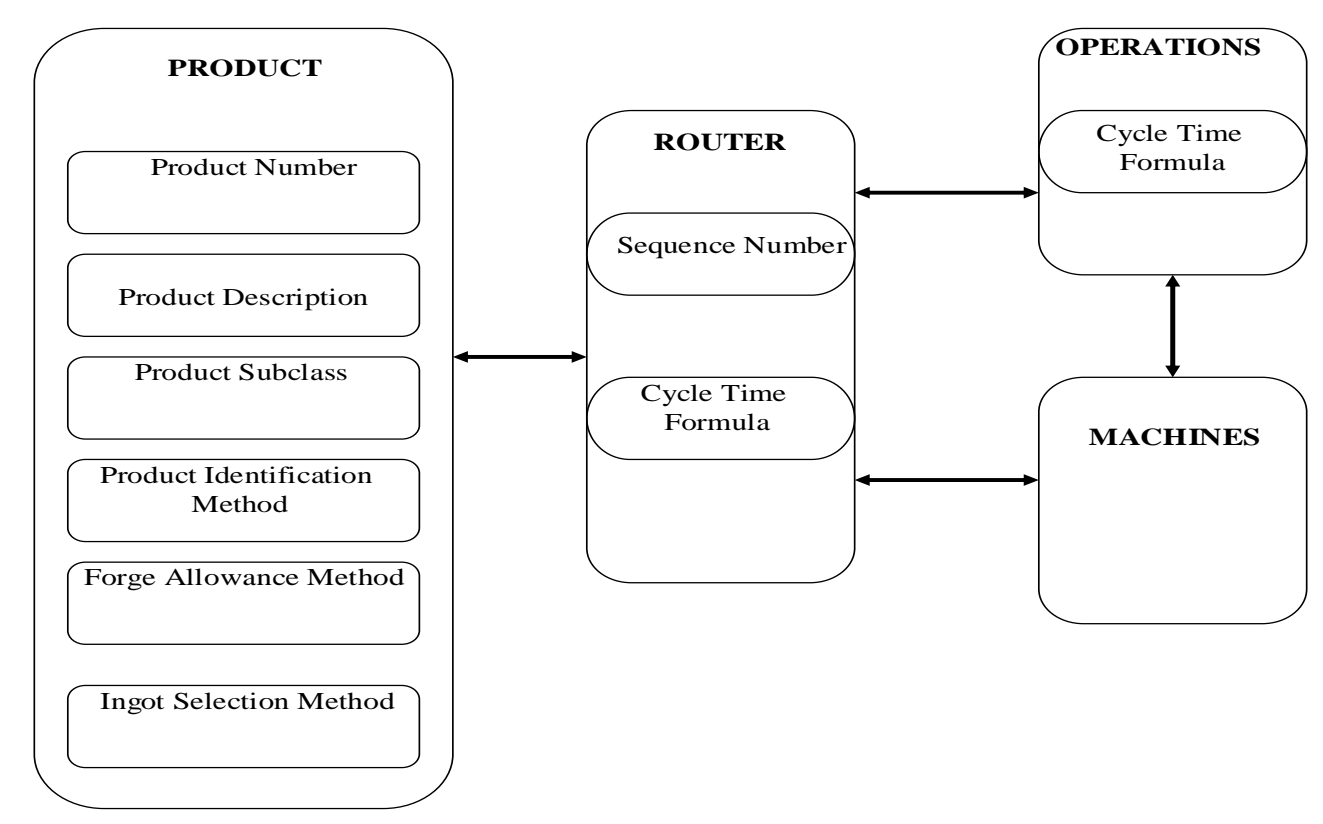

Figure 5

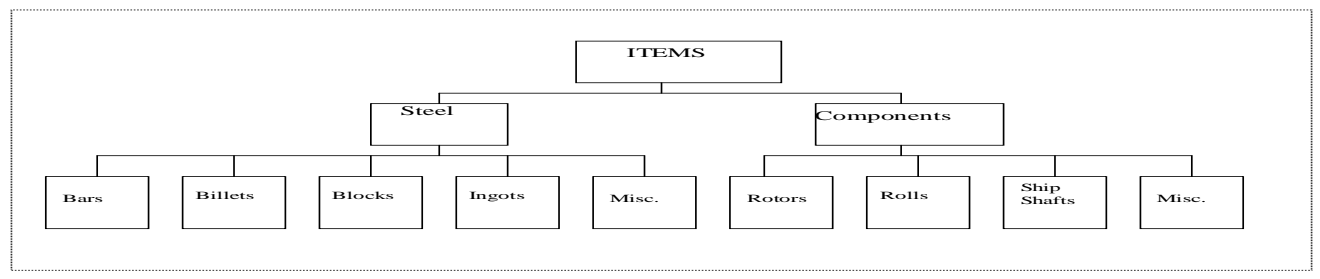

This approach provides efficient means to acquire the sales and other related information. It defines the product very precisely in terms both the customers and sales people understand. This also limits the number of potential product types that is small enough to be exhaustively searched. The role of the decision support system at this stage is to ask the appropriate questions needed for the required inputs from the sales staff and to assist them in better expressing the customer's requirements. The decision support system also minimizes any redundancies involved in the user inputs, yet captures all the necessary information. The graphical user interface makes the system user friendly.

Once the product feature set is expressed by the salesperson the decision support system will access the relevant knowledge and carry out the bidding process. This determination begins by finding product number, a 
classification by shape and generic manufacturing processing requirements. The user enters the product feature set and geometry. The system identifies the underlying generic product and applies the relevant procedures employed to compute the forging allowances and select the appropriate ingot. Once an ingot is chosen the steel costs can be estimated using the standard "steel estimation" procedure. After calculating the material costs, the system accesses the appropriate routers needed to manufacture the product. Each of the operations on the router has a unique cycle time formula. To process the formula, system should be able to successfully provide data for all the variables used in the cycle time formula. After the cycle times have been computed, the manufacturing cost for each operation is determined and overall manufacturing cost of the product is calculated. This enables the system to establish the standard cost for that product.

The inference engine has the ability to uncover any corruption and inadequacies in the knowledge base. Typical situations include product types with overlapping characteristics or new products that have not yet been defined. The system deals with these situations by assigning a "99999" generic product number and informs the sales staff that the customer has requested a new type of product that requires costing by the engineering staff. This is a built in feature that provides a graceful exit for the system.

The last step in the bidding process is determination of the profit margins. Each product may have a different profit margin based on the complexity of the manufacturing processes required, tests conducted, materials used, market conditions, capacity constraints, and internal utilization rates. The expertise to administer this step lies with the senior management. The determination of profit margin, as already mentioned, then becomes straightforward addition of certain percentage, suggestion to consult the engineers or sales management, or asking the user to input the appropriate value. A rule base is used to capture this expertise and the system assigns the appropriate profit margin rates based on the above set of conditions.

\subsection{Testing and Validation Issues}

In the testing phase, the system was enthusiastically received. The engineering staff and salespersons used the system and indicated that the system was useful, easy to update, and user friendly. This decision support system is knowledge based and validation of the system can only be done by engineers and salespersons. The validation of the decision support system was done in two stages. In stage 1, two experienced engineers were chosen as experts and completed a bid using the system. The engineers completed the task accurately for the existing product in 35 minutes as opposed to 8 hours in the earlier system. For a non-existent but similar product similar time reductions were recorded, though the time saved would vary as per complexity of the product. In stage 2 , the novice (salesperson) was asked to complete the bid and successfully completed the task in one hour for an existing product. Again, for a non-existent but similar product, the salesperson can narrow down the possibilities and send the specifications to engineers much quicker than earlier system. Here also results would vary according to the complexity of the product. However, the validation of the entire procedural and declarative knowledge base would have taken too much engineers' time and was deemed infeasible.

The initial testing phase was successful and well received. In the medium-term we faced following problems for acceptance and validation of the system:

- User involvement: The engineers and salespersons were involved in designing and developing of the system. However, once the knowledge engineering was complete the system was built in isolation. The engineers felt that they were dealing with the black box, although, they were able to update the system. Additionally, if the updating involved rewriting of VBA code they had to turn to programmers and the company did not have in-house programmers. As the system was updated it would necessitate another round of validation. The workload of engineers also quickly forced them to fall into old habits and updating of the system became an issue.

- $\quad$ Explanation facilities: Since the knowledge base is not entirely rule driven, we did not deem explanation facilities as an important part of the system. The chosen methodology also was not conducive to providing explanation facilities. The acceptance of the system might have been easier if explanation facilities were used. 
- $\quad$ Programmer turnover: The VBA programs were written by teams of students. The student teams performed admirably well, however, the project lasted for about two years. There was a lot of turnover in two years and we had problems in tracking down program changes, updates, and debugging problems.

- Lack of documentation: Another aspect of programmer turnover was lack of adequate documentation. As the system became more complex, this problem became more acute. We tried hard to organize the documentation according to versions of the system, but we faced serious problems in user manuals, program codes, changes, and updates.

We tracked the usage of the system for many months. However, the backlog in terms of updating the system, user education, and financial difficulties of the company undid the use of the system.

\subsection{Conclusions}

This paper has presented a novel approach in integrating knowledge-based technologies with the relational database technologies. It makes the use of group technology coding schemes in identifying the parts based on the feature set, processing requirements, and other business factors. It explains the design methodology used in developing the knowledge based bidding system. We have shown successfully how knowledge based and objectoriented principles can be used in building a transaction centered database system. Systems of this kind are critical for the success of a job order business. They not only lower operational costs but also provide the critical knowledge needed to evaluate the costs associated with the order. Ideally, the system's objective is to estimate the costs closer to the actual costs. Another advantage of this system is to do "what-if" analysis. What if I buy a new type of forging machine or a lathe? What benefits can the business realize based on what the orders were for the time period? It also provides essential feedback needed to explain why the business was unable to grab a specific order. The system has the potential to promote standards and impose discipline among technical engineers and the sales staff. We, however, underestimated the complexities involved in designing, maintaining, and selling the system to the engineering staff. These complexities and financial difficulties encountered by the company undermined success of the system.

\subsection{Suggestions for future research}

Bidding for customized job orders is a classic as well as a current problem. This problem exists in a variety of industries. For example, this framework can be easily extended to other industries such as health care. For instance, for a health care provider such as a hospital, a patient's visit for service can be treated as job order. All the services provided for a patient need to be cost accurately. A hospital has all the needed expertise to classify a patient into generic patient classifications. Such extensions will open new applications of group technology, and innovative decision support systems.

\section{References}

1. Ben-Arieh, D., S. Lee, and P. Chang, 1996, "Fuzzy part coding for Group Technology," European Journal of Operational Research, Vol. 92, pp. 637-648.

2. Billo, R. and B. Bidanda, 1995, "Representing group technology classification and coding techniques with object oriented modeling principles," IIE Transactions, Vol. 27, pp. 542-554.

3. Cheng, C. H., C. Goh, and A. Lee, 2001, "Designing group technology manufacturing systems using heuristics branching rules," Computers and Industrial Engineering, Vol. 40, pp. 117-131.

4. Djassemi, M., 2000, "An efficient CNC programming approach based on group technology," Journal of Manufacturing Systems, Vol. 19, pp. 213-217.

5. Dowlatshai, S, and M. Nagraj, 1998, "Application of group technology for design data management," Computers and Industrial Engineering, Vol. 34, pp. 235-255.

6. Edwards, J. S., Y. Duan, and P. C. Robbins, 2000, "An analysis of expert systems for business decision making at different levels and in different roles," European Journal of Information Systems, Vol. 9, No. 1, pp. 36-46. 
7. Gunasekaran, A., S. Goyal, I. Virtanen, and P. Yli-Olli, 1994, "An investigation into the application of group technology in advanced manufacturing systems," International Journal of Computer Integrated Manufacturing, Vol. 7, pp. 215-228.

8. Henderson, M., 1984, Extraction of feature information from three-dimensional CAD data, Unpublished Ph.D. Dissertation, School of Mechanical Engineering, Purdue University, West Lafayette, Indiana.

9. Jakubowski, R., 1982, "Syntactic characterization of machined part shapes," Cybernetics, Vol. 13, pp. 1-24.

10. Kingsman, B. and A. De Souza, 1997, "A knowledge-based support system for cost estimation and pricing decisions in versatile manufacturing companies," International Journal of Production Economics, Vol. 53, pp. 119-139.

11. Kuo, R. J., C. Chi, and P. W. Teng, 2001, “Generalized part family formation through fuzzy self-organizing feature map neural network," Computers and Industrial Engineering, Vol. 40, pp. 79-100.

12. Li, R., 1988, "A part-feature recognition system for rotational parts," International Journal of Production Research, Vol. 26, pp. 1451-1475.

13. Keefe, R., and A. Preece, 1996, "The development, validation, and implementation of knowledge-based systems," European Journal of Operational Research, Vol. 92, Issue 3, pp. 458-474.

14. Vakharia, A, 1986, "Methods of cell formation in group technology: A framework for evaluation," Journal of Operations Management, Vol. 6, pp. 257-271.

15. Wemmerlov, U. and N. Hyer, 1986, "Procedures for the part/family group identification problem in cellular manufacturing," Journal of Operations Management, Vol. 6, pp. 125-147. 
Notes 